\title{
Comparison of Optical Surface Guide System and ConeBeamCT Scans in Patient Positioning in Breast Cancer Radiotherapy
}

\section{Meme kanseri Radyoterapisinde Hasta Pozisyonlandırılmasında Optik Yüzey Takip Sistemi ve ConeBeamCT Taramalarının Karşılaştırılması}

\author{
İsmail Faruk Durmuș, Bora Taş \\ İstanbul Yeni Yüzyıl Üniversitesi Özel Gaziosmanpaşa Tıp Fakültesi Hastanesi \\ Radyasyon Onkolojisi Bölümü, İstanbul
}

Dergiye Ulaşma Tarihi: 20.01.2019 Dergiye Kabul Tarihi:01.03.2019 Doi: 10.5505/aot.2019.83584

\section{ÖZET}

GiRIŞ ve AMAÇ: Meme Radyoterapisinde tedavinin başarısı, hedef volümün doğru ışınlamasına ve hastanın doğru konumlandırılmasına bağlıdır. Lazer tabanlı optik yüzey takip sisteminin meme Radyoterapisinde hasta pozisyonlandırma ve tedavinin her fraksiyonda tekrarlanabilirliği araştırıldı. Bu sayede hastaya portal görüntüleme amaçlı uygulanan radyasyonun azaltılması da araştıııldı.

YÖNTEM ve GEREÇLER: Catalyst optik yüzey takip sistemi kullanılarak 3D yüzey görüntüleme ile meme kanserli hastalarda pozisyonlandırma yapıldı, ardından $\mathrm{kV}$ tabanlı CBCT ile taramalar yapılarak iki sistem arasında hasta pozisyonunda olan sapmalar belirlendi. 26 Meme kanserli hastaya 475 fraksiyonda lazer tabanlı optik yüzey ve CBCT taramaları yapıldı. Her bir hastanın ayrı ayrı ve 475 fraksiyonun tamamı için, ortalama ve maksimum sapma miktarları lateral, longitudinal ve vertikal eksenlerde bulundu.

BULGULAR: 475 Fraksiyonda Catalyst ve CBCT değerleri arasındaki ortalama farklar lateral eksende; $0.24 \pm 0.22 \mathrm{~cm}$, longitudinal eksende; $0.36 \pm 0.28 \mathrm{~cm}$, vertikal eksende; $0.22 \pm 0.20 \mathrm{~cm}$ bulundu. Catalyst ile CBCT değerleri arasındaki maksimum farklar lateral eksende; $1.28 \mathrm{~cm}$, longitudinal eksende; $1.54 \mathrm{~cm}$, vertikal eksende; $1.30 \mathrm{~cm}$ bulundu. Eğer 26 hasta için sadece Catalyst yüzey takip sistemi kullanılmış olsaydı, klinik hedef volüme $5 \mathrm{~mm}$ emniyet marji verilirse lateral eksende $\% 89.05$, longitudinal eksende $\% 74.52$ ve vertikal eksende $\% 90.52$ güven aralığı içinde tedavi uygulanabilmektedir.

TARTIŞMA ve SONUÇ: Meme Radyoterapisinde tedavinin tekrarlanabilirliği ve hasta konumlandırmasının kesin hassasiyetle olması büyük öneme sahiptir. Optik yüzey takip sistemi hasta setup'ı için basit, hızlı, tekrarlanabilir ve doğru bir çözümdür. Rastgele günlük setup hatalarını en aza indirmek ve hastaya tedavi harici portal görüntüleme amaçlı uygulanan radyasyonu azaltmak için etkin kullanılabilmektedir. Hasta tedaviye başladığı ilk birkaç fraksiyon CBCT ve optik yüzey takip sistemi eş zamanlı kullanılmalı aradaki farkların az olması durumunda, hastanın tedavisinin tamamı göz önüne alınarak CBCT sayısı azaltılıp optik yüzey takip sistemi kullanılmalıdır.

Anahtar Kelimeler: Yüzey kılavuzlu Radyoterapi, Meme IGRT, ConeBeamCT

ABSTRACT

INTRODUCTION: The success of treatment in breast radiotherapy depends on the correct irradiation of the target volume and correct positioning of patient. Patient positioning and repeatability of treatment at each fraction were investigated. In this way, the reduction of radiation implemented to the patient for portal imaging was also investigated.

METHODS: Positioning in breast cancer patients with 3D surface imaging was performed using the Catalyst optical surface guide system. Then, scans with $\mathrm{kV}$-based CBCT were used to determine deviations in the patient position between the two systems. The laser-based optical surface and CBCT scans were performed in 475 fractions.

RESULTS: The mean and maximum deviation amounts for each patient and all of the 475 fractions were obtained in lateral, longitudinal and vertical axes. The mean differences between Catalyst and CBCT values in the 475 fraction were $0.24 \pm 0.22 \mathrm{~cm}$ in the lateral axis, $0.36 \pm 0.28 \mathrm{~cm}$ in the longitudinal axis and $0.22 \pm 0.20 \mathrm{~cm}$ in the vertical axis. The maximum differences between Catalyst and CBCT values were obtained $1.28 \mathrm{~cm}$ in lateral axis, $1.54 \mathrm{~cm}$ in longitudinal axis and $1.30 \mathrm{~cm}$ in vertical axis. If only the Catalyst was used for 26 patients, a margin of $5 \mathrm{~mm}$ was implemented to clinical target volume, treatment could be performed in lateral axis at $89.05 \%$, in longitudinal axis $74.52 \%$ and in vertical axis at $90.52 \%$ confidence interval. 
DISCUSSION AND CONCLUSION: Reproducibility of the treatment and accurate positioning of the patient are very important in breast radiotherapy. Catalyst system is a simple, fast, repeatable and accurate solution for patient setup. It can be used effectively to minimize random daily setup errors and reduce radiation implemented to the patient for external portal imaging. CBCT and Catalyst should be used simultaneously in the first few fractions. If the deviations between the two systems are low, the number of CBCT should be reduced, taking into account the full treatment of the patient.

Keywords: Surface Guided Radiotherapy, Breast IGRT, ConeBeamCT

\section{GíRiş}

Radyoterapi de amaç tanımlanmış hedef hacme optimum doz verilirken, hedefin etrafindaki sağlıklı doku ve organları mümkün olduğunca korumaktır. Sağlıklı doku korumasını arttırmak ve hedef hacimde daha konformal doz dağılımı elde etmek için, Intensity Modulated Radiotherapy (IMRT) ve Volumetric Modulated Arc Therapy (VMAT) gibi teknikler geliştirilmiştir. Bu teknikler ile daha konformal doz dağılımı ve hedef dışında hızlı bir doz düşüşü sağlanmaktadır. $\mathrm{Bu}$ nedenle hastanın pozisyon doğruluğu ve hedefin 1skalanmaması kritik öneme sahiptir. Hastanın tedavisi süresince her fraksiyonda hastanın doğru pozisyonlandırılması hem hedefin planlanan dozu almasını sağlamakta, hem de sağlıklı organ dozlarını mümkün mertebe azaltacağı için radyasyona bağlı toksisiteyi azaltılabilmektedir. $(1,2)$. Bu nedenle tedavi tekrarlana bilirliğinin doğruluğunun geliştirilmesi, lokal kontrol oranını arttırabilmekte ve radyasyona bağlı komplikasyonları da azaltabilmektedir. (3) Image Guide Radyoterapi (IGRT) teknikleri ile hastanın pozisyonu ve hedefin doğru 1şınlanması sağlanmaktadır. Özellikle tedavi öncesinde ConeBeamCT (CBCT) taramalarıyla tedavinin setup doğruluğunu yüksek seviyede sağlanabilmektedir. Fakat bu esnada hastaya tedavi harici ek radyasyon uygulanmaktadır. Ayrıca CBCT sadece tedavi öncesi hasta pozisyonu ile ilgili bilgi içerdiğinden tedavi esnasında olabilecek hareket hakkında bilgi içermemektedir.(4,5) Hastaya ek radyasyon uygulamadan diğer IGRT teknikleri ultrason ve optik yüzey takip sistemleridir. $(6,7,8)$. Bu sistemlerle tedaviler arası (inter-fraction) ve tedavi esnasinda (intrafraction) takip yapilabilmektedir. $\mathrm{Bu}$ sistemlerin en büyük avantajı non-invaziv ve tedavi harici radyasyon uygulanmadan takip yapılabilmeleridir. Optik yüzey takip sistemleri ile sadece hastanın yüzeyi takip edilebilmektedir. Daha derin yerleşimli hedef veya dokuların takip edilen yüzey ile arasındaki korelasyonuna bağlı olması bu sistemlerin sınırlandırıcı faktörüdür. (9)

$$
\text { Çalışmamızın amacı meme }
$$

Radyoterapisinde Catalyst ${ }^{\mathrm{TM}}$ (C-RAD, AB, Uppsala, Sweden) optik yüzey takip sistemini ve CBCT taramalarını kullanarak hastanın ve hedefin doğru pozisyonda olduğunu analiz etmek ve bu iki sistem arasındaki korelasyonu belirlemektir.

\section{GEREÇ ve YÖNTEM}

26 Meme kanserli hastasina supine pozisyonda alpha-cradle (wingSTEPTM, Elekta, Austria) sabitleme ekipmanı ile belirli bir açıda eğimli olarak pozisyonlandırıldı. Her bir hasta için $120 \mathrm{kVp}, \quad 75 \mathrm{mAs}$ değerlerinde Siemens ${ }^{\circledR}$ Biograph mCT (Knoxville, TN, USA) cihazı ile $3 \mathrm{~mm}$ kesit aralıklıtaramalar yapıldı. Referans noktaları belirlemek için klasik lazer sistemi kullanıldı. Monaco 5.11 (Elekta AB, Stockholm, Sweden) tedavi planlama sisteminde hedef ve riskli organlar konturland. Hedef ve kritik organlara göre VMAT, IMRT tekniği kullanılarak planlar hazırlandı. Planları hazırlanan $\mathrm{CT}$ veri setleri Catalyst ${ }^{\mathrm{TM}}$ optik yüzey takip sistemine aktarıldı. Sağ veya sol meme olmasina bağlı olarak yüzey tarama alanı için ilgilenilen bölge belirlendi. Elekta Versa HD(Elekta AB, Stockholm, Sweden) lineer hizlandırıcisinda tedavi öncesinde hastalar tedavi masasına yatırıldıktan sonra lazerlerle referans noktalara göre pozisyonlandirıldı. Daha sonra izosantr noktasına göre gerekli masa kaydırmaları yapılarak hasta pozisyonlandırıldı. Ardından hastaların $\mathrm{XVI}^{\circledR}$ 5.0 IGRT sistemi ile CBCT taramaları gerçekleştirildi.

Catalyst Yüzey Tarama Sistemi: Optik yüzey takip sistemi olan Catalyst ${ }^{\mathrm{TM}}$ (C-RAD $\mathrm{AB}$, Sweden); Light-emitting diode (LED) ve Charge Coupled Device (CCD) kamera sistemi olan iki komponentten oluşur. Tedavi masasının ayak tarafina tavana monte edilir. 
Hastanın cildini taramak için, yakın görünür bölgede elektromanyetik dalga gönderip sonra hastanın cildinden yansıyan ışı kamera tarafindan toplanır. Hastanın pozisyonu kamera tarafindan toplanan 1şı̆̆ın optik üçgenleme mantığıyla hesaplaması sonucu belirlenir. Catalyst ${ }^{\mathrm{TM}}$ sisteminin özellikleri; frekansı $47-63 \mathrm{~Hz}$, tarama 1şığının dalga boyu $405 \mathrm{~nm}$ (mavi), $528 \mathrm{~nm}$ (yeşil), $624 \mathrm{~nm}$ (kırmızı) dır. Tarama alanı; $800 \mathrm{~mm}$ x 1300 $\mathrm{mm} \quad \mathrm{x} \quad 700 \mathrm{~mm} \quad(\mathrm{X}, \mathrm{Y}, \mathrm{Z})$. Ölçüm tekrarlanabilirliği; $\quad 0.2 \mathrm{~mm}$. Pozisyon doğruluğu 1 mm'den daha küçüktür $(10,11,12)$.

Hastaların tedavisinde 475 fraksiyonda hem vücut yüzeyi takip sistemi hem de CBCT taramaları gerçekleştirildi. Lateral, longitudinal ve vertikal eksende yüzey takip sistemi ile CBCT arasındaki farklar bulundu. Hastaların her biri için ayrı ayrı ve 475 fraksiyonun tamamı için ortalama ve maksimum değerler bulundu. Standart sapmaları hesaplandı.

Catalyst ve CBCT arasindaki lateral, longitudinal ve vertikal eksendeki farklar, 475 fraksiyon için grafik 1, grafik 2 ve grafik 3 de gösterilmiştir.

Optik yüzey takip sistemi ile CBCT taramalarını arasındaki farklar değerlendirildiğinde, en fazla $\pm 0.1 \mathrm{~cm}$ farkın olması lateral yönde \%33.68, longitudinal yönde $\% 20.84$, vertikal yönde $\% 34.31$ güven aralığ 1 içinde bulundu. Maksimum $\pm 0.3 \mathrm{~cm}$ farkın olması lateral yönde \%71.78, longitudinal yönde $\% 53.05$ ve vertikal yönde \%77.26 güven aralığı içinde bulundu. Maksimum $\pm 0.5 \mathrm{~cm}$ farkın olması lateral yönde $\% 89.05$, longitudinal yönde $\% 74.52$ ve vertikal yönde $\% 90.52$ güven aralığ 1 içinde bulundu. Maksimum $\pm 1 \mathrm{~cm}$ farkın olmas1 lateral yönde \%98.73, longitudinal yönde $\% 97.26$ ve vertikal yönde \%99.36 güven aralığı içinde bulundu. Tablo 2 de $\pm 0.1 \mathrm{~cm}$, $\pm 0.1 \mathrm{~cm}$ ile $\pm 0.3 \mathrm{~cm}$ aralığında, $\pm 0.3 \mathrm{~cm}$ ile $\pm 0.5 \mathrm{~cm}$ aralığında, $\pm 0.5 \mathrm{~cm}$ ile $\pm 1 \mathrm{~cm}$ aralığında Catalyst ve CBCT arasında sapmaların kaç fraksiyonda olduğu gösterilmektedir.

\section{TARTIŞMA}

Catalyst optik yüzey takip sistemini kullanarak 3D yüzey görüntülemenin meme kanserli hastalarında olabilecek pozisyonlandırma hatalarını, CBCT tabanlı görüntü kılavuzluğuna göre karşılaştırarak oluşabilecek sapmaları analiz edildi.

Walter ve ark. 25 hastada 154 fraksiyon boyunca optik yüzey takip sistemi ile CBCT taramalarını karşılaştırmışlardır. Hastalar pelvik, abdominal ve toraks bölgesi olarak seçmişlerdir. Toraks bölgesi için lateral eksende $0.6 \pm 2.6 \mathrm{~mm}$, longitudinal eksende $5 \pm 7.9 \mathrm{~mm}$, vertikal eksende $0.5 \pm 3.2 \mathrm{~mm}$ optik yüzey tarama ile CBCT arasinda fark bulmuşlardır. Abdomen bölgesi için lateral eksende $0.3 \pm 2.2 \mathrm{~mm}$, longitudinal eksende $2.6 \pm 1.8 \mathrm{~mm}$, vertikal eksende $2.1 \pm 5.5 \mathrm{~mm}$ optik yüzey tarama ile CBCT arasında fark bulmuşlardır. Pelvis bölgesi için lateral eksende $-0.9 \pm 1.5 \mathrm{~mm}$, longitudinal eksende $1.7 \pm 2.8 \mathrm{~mm}$, vertikal eksende $1.6 \pm 2.2 \mathrm{~mm}$ optik yüzey tarama ve CBCT arasında fark bulmuşlardır. Bizim çalışmamız olduğu gibi longitudinal eksende en büyük farklar bulunmuştur. Derin yerleşimli tümörlerde cilt ile hedef arasındaki hareketin korelasyonu farkların değişkenliğine yol açabilmektedir. (10)

Ma ve ark. Meme kanserli 20 hastada 200 fraksiyonda CBCT ve optik yüzey tarama sistemi arasındaki korelasyonu analiz etmişlerdir. Yüzey takip sistemi ve CBCT arasındaki farklar lateral eksende $0.008 \mathrm{~cm}$, longitudinal eksende $0.022 \mathrm{~cm}$ ve vertikal eksende $0.003 \mathrm{~cm}$ olarak bulmuşlardır. En yüksek sapmayı longitudinal yönde bulmuşlardır. Çalışmamız ile uyumludur. (3)

Crop ve ark. Meme kanserli hastaların tedavi öncesi Helical Tomoterapi cihazında MV-CT ve Catalyst yüzey takip sistemi ile pozisyonlandırıp karşılaştırmışlardır. İki IGRT tekniği arasında sağ-sol, cranio-caudal ve anterio-posterior yönlerde sirasiyla $0.24 \mathrm{~cm}$, $0.27 \mathrm{~cm}$ ve $0.24 \mathrm{~cm}$ fark bulmuşlardır. $\mathrm{Bu}$ sonuçlar doğrultusunda kliniklerinde ilk 5 fraksiyon her iki IGRT tekniğini kullanmışlar daha sonraki fraksiyonlarda her hasta için klinik değerlendirme yapılarak MV-CT sayısını azaltmışlardır. (13)

Analizini gerçekleştirdiğimiz 26 hasta için sadece Catalyst yüzey takip sistemi kullanılmış olsaydı klinik hedef volüme emniyet $3 \mathrm{~mm}$ marj verilmesi durumunda lateral yönde $\% 71.78$, longitudinal yönde $\% 53.05$ ve vertikal yönde $\% 77.26$ güven aralığ 1 içinde tedavi uygulanabilmektedir. Eğer 
klinik hedef volüme $5 \mathrm{~mm}$ emniyet marj1 verilirse lateral yönde \%89.05, longitudinal yönde $\% 74.52$ ve vertikal yönde $\% 90.52$ güven aralığı içinde tedavi uygulanmaktadır.

Meme Radyoterapisinde, tedavinin başarısı hedef volümün doğru 1şınlamaktan geçmektedir, hastanın doğru konumlandırılması büyük öneme sahiptir. $\mathrm{Bu}$ IGRT işlemini yaparken hastaya portal amaçlı uygulanan radyasyon dozunu azaltmak için lazer tabanlı yüzey takip sistemi kullanılabilmektedir. Hasta tedaviye başladığı ilk birkaç fraksiyon CBCT ve optik yüzey takip sistemi eş zamanlı kullanılmalı aradaki farkların az olmasi durumunda, hastanın tedavisinin tamamı göz önüne alınarak CBCT say1s1 azaltılıp optik yüzey takip sistemi kullanılmalıdır. Ayrica Meme Radyoterapisinde bu tür çalışmalar az olduğu için yüzey takip sistemlerinin uygulanması için yol gösterici bir çalışmadır.

\section{SONUÇ}

26 Hastanın toplamda 475 fraksiyonda Catalyst ve CBCT değerleri arasındaki ortalama fark, lateral eksende (X); $0.24 \pm 0.22$ $\mathrm{cm}$, longitudinal eksende $(\mathrm{Y}) ; 0.36 \pm 0.28 \mathrm{~cm}$, vertikal eksende $(\mathrm{Z}) ; 0.22 \pm 0.20 \mathrm{~cm}$ bulundu. Catalyst ile CBCT kaydırma değerleri arasındaki maksimum fark, lateral eksende $(\mathrm{X}) ; 1.28 \mathrm{~cm}$, longitudinal eksende (Y); 1.54 $\mathrm{cm}$, vertikal eksende $(\mathrm{Z}) ; 1.30 \mathrm{~cm}$ bulundu. Tablo 1 de Catalyst ve CBCT taramaları arasındaki farklar 26 hasta için ayrı ayrı ve 475 fraksiyonun tamamı için gösterilmektedir.

Çıkar çatışması: Yok

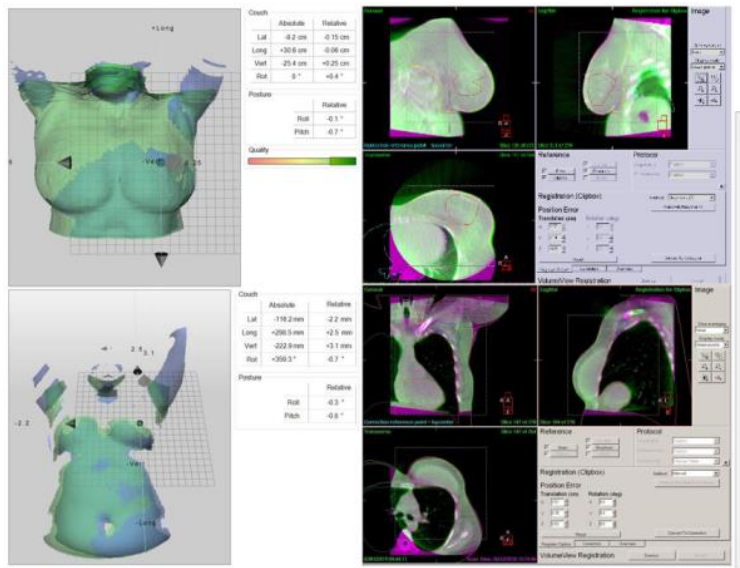

Şekil 1: Catalyst ve XVI hasta tarama görüntüleri

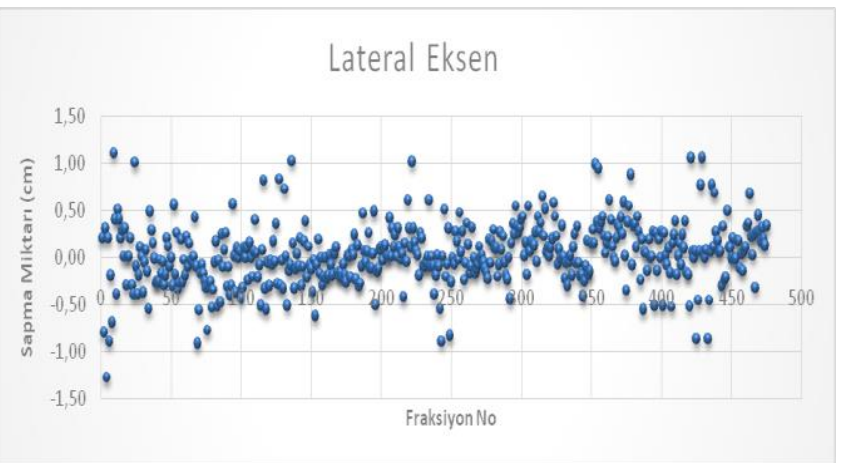

Grafik 1: 475 Fraksiyonda lateral eksende Catalyst ve CBCT arasındaki sapmalar

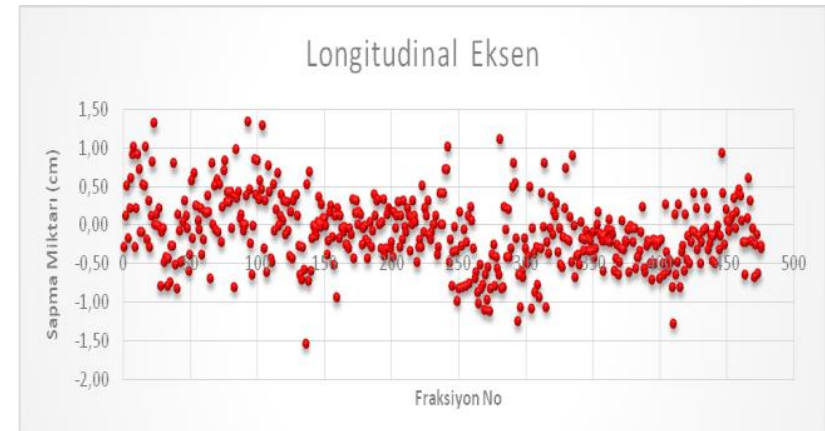

Grafik 2: 475 Fraksiyonda longitudinal eksende Catalyst ve $\mathrm{CBCT}$ arasındaki sapmalar

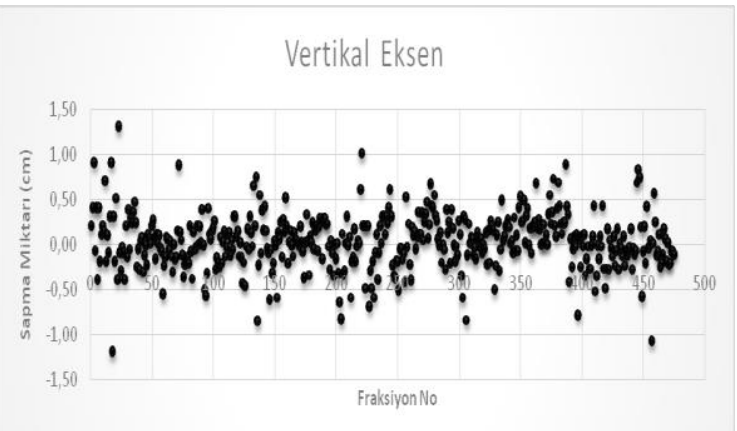

Grafik 3. 475 Fraksiyonda vertikal eksende Catalyst ve CBCT arasındaki sapmalar 
Tablo 1: CBCT ve Catalyst ${ }^{\mathrm{TM}}$ arasındaki lateral, longitudinal, vertikal eksendeki farklar

\begin{tabular}{|c|c|c|c|c|c|c|c|}
\hline & \multirow[b]{2}{*}{ Fraksiyon sayısı } & \multicolumn{3}{|c|}{ Ortalama Fark } & \multicolumn{3}{|c|}{ Maksimum Fark } \\
\hline & & $\mathrm{X}(\mathbf{c m})$ & $\mathbf{Y}(\mathbf{c m})$ & $\mathbf{Z}(\mathbf{c m})$ & $X(\mathbf{c m})$ & $\mathbf{Y}(\mathbf{c m})$ & $\mathbf{Z}(\mathbf{c m})$ \\
\hline Hasta 1 & 9 & 0.63 & 0.45 & 0.34 & 1.28 & 1.00 & 0.90 \\
\hline Hasta 2 & 20 & 0.27 & 0.41 & 0.38 & 1.00 & 1.30 & 1.30 \\
\hline Hasta 3 & 14 & 0.23 & 0.45 & 0.22 & 0.55 & 0.83 & 0.46 \\
\hline Hasta 4 & 24 & 0.19 & 0.29 & 0.13 & 0.92 & 0.78 & 0.56 \\
\hline Hasta 5 & 25 & 0.31 & 0.35 & 0.18 & 0.92 & 1.32 & 0.87 \\
\hline Hasta 6 & 26 & 0.23 & 0.48 & 0.19 & 0.81 & 1.32 & 0.58 \\
\hline Hasta 7 & 13 & 0.27 & 0.25 & 0.17 & 0.82 & 0.72 & 0.64 \\
\hline Hasta 8 & 28 & 0.22 & 0.37 & 0.28 & 1.02 & 1.54 & 0.86 \\
\hline Hasta 9 & 26 & 0.14 & 0.18 & 0.12 & 0.32 & 0.45 & 0.38 \\
\hline Hasta 10 & 21 & 0.16 & 0.22 & 0.24 & 0.51 & 0.42 & 0.84 \\
\hline Hasta 11 & 12 & 0.14 & 0.15 & 0.17 & 0.60 & 0.50 & 0.60 \\
\hline Hasta 12 & 17 & 0.24 & 0.20 & 0.34 & 1.01 & 0.50 & 1.00 \\
\hline Hasta 13 & 10 & 0.28 & 0.47 & 0.27 & 0.90 & 1.00 & 0.60 \\
\hline Hasta 14 & 17 & 0.23 & 0.41 & 0.25 & 0.84 & 0.99 & 0.52 \\
\hline Hasta 15 & 21 & 0.24 & 0.33 & 0.23 & 1.28 & 1.54 & 1.30 \\
\hline Hasta 16 & 4 & 0.13 & 0.75 & 0.25 & 0.31 & 1.13 & 0.66 \\
\hline Hasta 17 & 23 & 0.16 & 0.23 & 0.09 & 0.26 & 0.43 & 0.21 \\
\hline Hasta 18 & 20 & 0.23 & 0.58 & 0.23 & 0.54 & 1.26 & 0.85 \\
\hline Hasta 19 & 18 & 0.23 & 0.34 & 0.15 & 0.64 & 1.07 & 0.51 \\
\hline Hasta 20 & 22 & 0.18 & 0.32 & 0.16 & 0.42 & 0.89 & 0.48 \\
\hline Hasta 21 & 19 & 0.31 & 0.26 & 0.23 & 0.98 & 0.65 & 0.67 \\
\hline Hasta 22 & 13 & 0.32 & 0.37 & 0.17 & 0.87 & 0.72 & 0.79 \\
\hline Hasta 23 & 18 & 0.20 & 0.50 & 0.24 & 0.73 & 1.28 & 0.30 \\
\hline Hasta 24 & 25 & 0.35 & 0.24 & 0.15 & 1.05 & 0.51 & 0.68 \\
\hline Hasta 25 & 15 & 0.13 & 0.25 & 0.35 & 0.62 & 0.92 & 1.08 \\
\hline Hasta 26 & 15 & 0.28 & 0.31 & 0.14 & 0.95 & 0.68 & 0.26 \\
\hline Toplam & 475 & 0.24 & 0.36 & 0,22 & 1.28 & 1.54 & 1.30 \\
\hline Std & & 0.22 & 0.28 & 0,2 & & & \\
\hline
\end{tabular}

Tablo 2: 475 Fraksiyon için 3 eksende sapma miktarlarına göre fraksiyon sayıları

\begin{tabular}{lccc}
\hline Sapma $(\mathbf{c m})$ & Lateral & Longitudinal & Vertikal \\
\hline $\mathbf{0 . 1}$ & 160 & 99 & 163 \\
$\mathbf{0 0 . 1}- \pm \mathbf{0 . 3}$ & 181 & 153 & 204 \\
$\mathbf{\mathbf { 0 . 3 }} \pm \mathbf{0 . 5}$ & 82 & 102 & 63 \\
$\pm \mathbf{0 . 5}- \pm \mathbf{1}$ & 46 & 108 & 42 \\
$> \pm \mathbf{1}$ & 6 & 13 & 3 \\
\hline \hline
\end{tabular}




\section{REFERANSLAR}

1. Ballivy O, Parker $\mathrm{W}$, Vuong $\mathrm{T}$, Shenouda $\mathrm{G}$, Patrocinio H. "Impact of geometric uncertainties, on dose distribution during intensity modulated radiotherapy of head-and-neck cancer:The need for a planning target volume and a planning organ-atrisk volume," Current Oncology, vol. 13, no. 3, pp. 108-115, 2006.

2. Krengli M, Gaiano M, Mones $\mathrm{E}$ et al., "Reproducibility of patient setup by surface image registration system in conformal radiotherapy of prostate cancer," Journal of Radiation Oncology, vol. 4, article no. 9, 2009.

3. Ma Z, Zhang W, Su Y, et al., "Optical Surface Management System for Patient Positioning in Interfractional Breast Cancer Radiotherapy," BioMed Research International, vol. 2018, Article ID 6415497, 8 pages, 2018. https://doi.org/10.1155/2018/6415497.

4. 1. Dawson LA, Sharpe MB. Image-guided radiotherapy: rationale, benefits, and limitations. Lancet Oncol. 2006;7:848-58.

5. Schneider U, Hälg R, Besserer J. Concept for quantifying the dose from image guided radiotherapy. Radia tOncol. 2015;10:188.
6. Li M, Ballhausen H, Hegemann NS, Ganswindt U, Manapov F, Tritschler S, Roosen A, Gratzke C, Reiner M, Belka C. A comparative assessment of prostate positioning guided by three-dimensional ultrasound and conebeamCT. Radiat Oncol. 2015;10:82.

7. Tas B, DurmusI.F,TokdemirOzturk S. Image Guided Radiotherapy (IGRT) Comparison between ConeBeamCT and Ultrasound Systemfor Prostate Cancer.UniversalJournal of Physicsand Application,2016;10:110 - $114 . \quad$ doi: 10.13189/ujpa.2016.100402.

8. Gaisberger C, Steininger P, Mitterlechner B, Huber $\mathrm{S}$, Weichenberger H, Sedlmayer F, Deutschmann $\mathrm{H}$. Three-dimensional surface scanning for acurcate patient positioning and monitoring during breast cancer radiotherapy. Strahlenther Onkol. 2013;189:887-980.

9. Wikström K, Nilsson K, Isacsson U, Ahnesjö A. A comparison of patient position displacements from body surface lasers canning and conebeamCT bone registrations for radiotherapy of pelvic targets. ActaOncol. 2014;53:268-77. 